\title{
A break in the gas and dust surface density of the disc around the T Tauri star IM Lupi
}

\author{
O. Panić ${ }^{1}$, M. R. Hogerheijde ${ }^{1}$, D. Wilner ${ }^{2}$, and C. $\mathrm{Qi}^{2}$ \\ ${ }^{1}$ Leiden Observatory, Leiden University, PO Box 9513, 2300 RA, Leiden, The Netherlands \\ e-mail: olja@strw.leidenuniv.nl \\ ${ }^{2}$ Harvard-Smithsonian Center for Astrophysics, 60 Garden Street, Cambridge, MA 02138, USA
}

Received 19 February 2009 / Accepted 2 April 2009

\begin{abstract}
Aims. We study the distribution and physical properties of molecular gas in the disc around the T Tauri star IM Lup on scales close to $200 \mathrm{AU}$. We investigate how well the gas and dust distributions compare and work towards a unified disc model that can explain both gas and dust emission.

Methods. ${ }^{12} \mathrm{CO},{ }^{13} \mathrm{CO}$, and $\mathrm{C}^{18} \mathrm{O} J=2-1$ line emission, as well as the dust continuum at $1.3 \mathrm{~mm}$, is observed at $1 . .8$ resolution towards IM Lup using the Submillimeter Array. A detailed disc model based on the dust emission is tested against these observations with the aid of a molecular excitation and radiative transfer code. Apparent discrepancies between the gas and dust distribution are investigated by adopting simple modifications to the existing model.

Results. The disc is seen at an inclination of $54^{\circ} \pm 3^{\circ}$ and is in Keplerian rotation around a $0.8-1.6 M_{\odot}$ star. The outer disc radius traced by molecular gas emission is $900 \mathrm{AU}$, while the dust continuum emission and scattered light images limit the amount of dust present beyond $400 \mathrm{AU}$ and are consistent with the existing model that assumes a $400 \mathrm{AU}$ radius. Our observations require a drastic density decrease close to $400 \mathrm{AU}$ with the vertical gas column density at $900 \mathrm{AU}$ in the range of $5 \times 10^{20}-10^{22} \mathrm{~cm}^{-2}$. We derive a gas-to-dust mass ratio of 100 or higher in disc regions beyond $400 \mathrm{AU}$. Within $400 \mathrm{AU}$ from the star our observations are consistent with a gas-to-dust ratio of 100 but other values are not ruled out.
\end{abstract}

Key words. planetary systems: protoplanetary disks - stars: individual: IM Lup - stars: pre-main sequence - circumstellar matter

\section{Introduction}

Low-mass star formation is commonly accompanied by the formation of a circumstellar disc. The disc inherits the angular momentum and composition of the star's parent cloud, and is shaped by the accretion and other physical processes within the disc during the evolution that may result in a planetary system. Over the past two decades observations of circumstellar discs at millimetre wavelengths have been established as powerful probes of the bulk of the cold molecular gas and dust. Spatially resolved observations of the molecular gas with (sub) millimetre interferometers constrain the disc size and inclination, the total amount of gas, its radial and vertical structure, and the gas kinematics (e.g., Guilloteau \& Dutrey 1998; Dartois et al. 2003; Qi et al. 2004; Isella et al. 2007; Piétu et al. 2007; Panić et al. 2008). In parallel, continuum observations of the dust at nearinfrared to millimetre wavelengths provide the disc spectral energy distribution (SED), that through modelling (e.g., Chiang \& Goldreich 1997; Dullemond et al. 2001; D’Alessio et al. 2005) yields the disc's density and temperature structure from the disc inner radius to a few hundred AU from the star. Studies of the gas through spatially resolved molecular line observations using results from the SED modelling (e.g., Raman et al. 2006; Panić et al. 2008) offer the means of addressing the gas-to-dust ratio, differences between the radial and vertical distributions of the gas and the dust, and the thermal coupling between the gas and the dust in the upper disc layers exposed to the stellar radiation (e.g., Jonkheid et al. 2004). Recent papers have suggested different disc sizes for the dust and the gas (e.g., HD 163296, Isella et al. 2007), which may be explained by sensitivity effects in discs with tapered outer density profiles (Hughes et al. 2008). Here, we present the results of a combined study using spatially resolved molecular-line observations and SED modelling of the disc around the low-mass pre-main-sequence star IM Lup.

Most pre-main-sequence stars with discs studied so far in detail are located in the nearby star-forming region of TaurusAurigae, accessible for the millimetre facilities in the northern hemisphere. Much less is known about discs in other starforming regions such as Lupus, Ophiuchus or Chamaeleon. It is important to compare discs between different regions, to investigate if and how different star-forming environments lead to differences in disc properties and the subsequent planetary systems that may result. IM Lup is a pre-main-sequence star located in the Lupus II cloud for which Wichmann et al. (1998) report a distance of $190 \pm 27$ pc using Hipparcos parallaxes. From its M0 spectral type and estimated bolometric luminosity of $1.3 \pm 0.3 L_{\odot}$, Hughes et al. (1994) derive a mass of $0.4 M_{\odot}$ and an age of $0.6 \mathrm{Myr}$ using evolutionary tracks from Swenson et al. (1994), or $0.3 M_{\odot}$ and 0.1 Myr adopting the tracks of D'Antona \& Mazzitelli (1994). In Pinte et al. (2008), a much higher value of $1 M_{\odot}$ is derived using tracks of Baraffe et al. (1998).

IM Lup is surrounded by a disc detected in scattered light with the Hubble Space Telescope (Pinte et al. 2008) and in the CO $J=3-2$ line with the James Clerk Maxwell Telescope by van Kempen et al. (2007). Lommen et al. (2007) conclude that grain growth up to millimetre sizes has occured from continuum measurements at 1.4 and $3.3 \mathrm{~mm}$. Recently, Pinte et al. (2008) present a detailed model for the disc around IM Lup based on the full SED, scattered light images at multiple wavelengths from the Hubble Space Telescope, near- and 
mid-infrared spectroscopy from the Spitzer Space Telescope, and continuum imaging at $1.3 \mathrm{~mm}$ with the Submillimeter Array. They conclude that the disc is relatively massive, $M \approx 0.1 M_{\odot}$ with an uncertainty by a factor of a few, has an outer dust radius not greater than $\approx 400$ AU set by the dark lane and lower reflection lobe seen in the scattered light images, and has a surface density $\Sigma(R)$ proportional to $R^{-1}$ constrained by the $1.3 \mathrm{~mm}$ data. Furthermore, they find evidence for vertical settling of larger grains by comparing the short-wavelength scattering properties to the grain-size constraints obtained at 1.4 and $3.3 \mathrm{~mm}$ by Lommen et al. (2007).

In this work, we present (Sect. 2) spatially resolved observations of the disc around IM Lup in ${ }^{12} \mathrm{CO},{ }^{13} \mathrm{CO}$ and $\mathrm{C}^{18} \mathrm{O}$ $J=2-1$ line emission, together with $1.3 \mathrm{~mm}$ dust continuum data, obtained with the Submillimeter Array ${ }^{1}$ (SMA). Our results (Sect. 3) show that the gas disc extends to a radius of 900 AU, more than twice the size inferred by Pinte et al. (2008). A detailed comparison (Sect. 4.1) to the model of Pinte et al. (2008) suggests a significant break in the surface density of both the gas and the dust around $400 \mathrm{AU}$, and we discuss possible explanations. We summarise our main conclusions in Sect. 5.

\section{Observations}

IM Lup was observed with the SMA on 2006 May 21 in a $8.6 \mathrm{~h}$ track, with a $4.3 \mathrm{~h}$ on-source integration time. The coordinates of the phase centre are $\mathrm{RA}=15^{\mathrm{h}} 56^{\mathrm{m}} 09^{\mathrm{s}} .17$ and $\mathrm{Dec}=$ $-37^{\circ} 56^{\prime} 06^{\prime \prime} 40$ (J2000). Eight antennas were included in an extended configuration providing a range of projected baselines of 7 to $140 \mathrm{~m}$. The primary beam half-power width is $55^{\prime \prime} 0$. The SMA receivers operate in a double-sideband (DSB) mode with an intermediate frequency band of $4-6 \mathrm{GHz}$ which is sent over fiber optic transmission lines to 24 "overlapping" digital correlator chunks covering a $2 \mathrm{GHz}$ spectral window in each sideband. The DSB system temperatures ranged from 90 to $150 \mathrm{~K}$. The correlator was configured to include the ${ }^{12} \mathrm{CO} J=2-1$ line $(230.5380000 \mathrm{GHz})$ in the upper sideband and the ${ }^{13} \mathrm{CO} 2-1$ $(220.3986765 \mathrm{GHz})$ and $\mathrm{C}^{18} \mathrm{O} 2-1$ line $(219.5603568 \mathrm{GHz})$ in the lower sideband. Each of the three lines was recorded in a spectral band consisting of 512 channels with $0.2 \mathrm{MHz}$ spacing $\left(\sim 0.26 \mathrm{~km} \mathrm{~s}^{-1}\right)$. Simultaneously to the line observations, the $1.3 \mathrm{~mm}$ dust continuum was recorded over a bandwidth of $1.8 \mathrm{GHz}$.

The data were calibrated and edited with the IDL-based MIR software package ${ }^{2}$. The bandpass response was determined from Jupiter, Callisto and 3C 273. After the passband calibration, broadband continuum in each sideband was generated by averaging the central $82 \mathrm{MHz}$ in all line-free chunks. Complex gain calibration was performed using the quasar J1626-298. The absolute flux scale was set using observations of Callisto. Subsequent data reduction and image analysis was carried out with the Miriad software package (Sault et al. 1995). The visibilities were Fourier transformed with natural weighting, resulting in a synthesized beam of $1^{\prime \prime} .8 \times 11^{\prime \prime} 2$ at a position angle of $0.2^{\circ}$. $1 \mathrm{Jy} /$ beam corresponds to $15.9 \mathrm{~K}$. The rms noise level is 125,94 and $102 \mathrm{mJy}^{\text {beam }}{ }^{-1}$ per channel respectively for the ${ }^{12} \mathrm{CO},{ }^{13} \mathrm{CO}$ and $\mathrm{C}^{18} \mathrm{O}$ spectral line data and $4 \mathrm{mJy}^{\text {beam }}{ }^{-1}$ for the continuum data.

\footnotetext{
${ }^{1}$ The Submillimeter Array is a joint project between the Smithsonian Astrophysical Observatory and the Academia Sinica Institute of Astronomy and Astrophysics and is funded by the Smithsonian Institution and the Academia Sinica.

${ }^{2}$ http://www.cfa.harvard.edu/ cqi/mircook.html
}

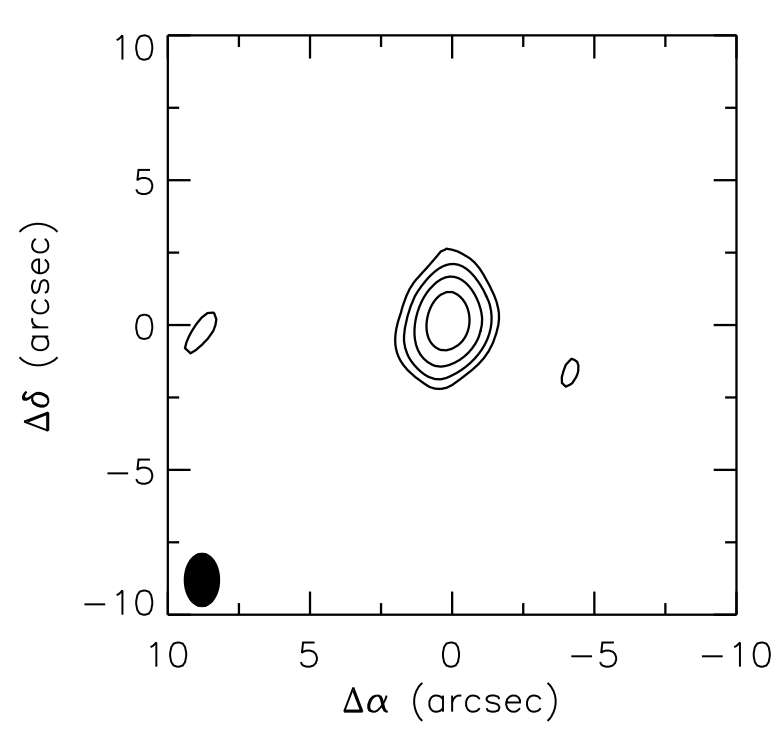

Fig. 1. Dust continuum image at $1.3 \mathrm{~mm}$. The contours are at $(2,4,8$, 16) $\times 3.67 \mathrm{mJy} \mathrm{beam}^{-1}(2,4,8,16$ sigma $)$ levels. The filled ellipse in the lower left corner shows the synthesized beam.

\section{Results}

\subsection{Dust continuum}

Figure 1 shows the $1.3 \mathrm{~mm}$ continuum emission observed toward IM Lup, previously reported in Pinte et al. (2008). The emission is centered on RA $=15^{\mathrm{d}} 56^{\mathrm{m}} 09^{\mathrm{s}} .20$, Dec $=-37^{\circ} 56^{\prime} 06^{\prime \prime} .5(\mathrm{~J} 2000)$, offset by $+00^{\prime} 4$ in right ascension and by $-0{ }^{\prime} .1$ in declination from the pointing center. We adopt the peak of the continuum emission as the position of IM Lup. The peak intensity of the continuum emission is $104 \pm 4 \mathrm{mJy}_{\text {beam }}{ }^{-1}$ and the total flux $176 \pm 4 \mathrm{mJy}$. The emission intensity is fit to the precision of one sigma by an elliptical Gaussian, yielding a source FWHM size of $1^{\prime \prime} .52 \pm 0$. .' $15 \times 11^{\prime \prime} .06 \pm 0$. . 15 and a position angle of $-35.5 \pm$ 4.0 deconvolved with the synthesized beam. This position angle, and the inclination in the range of $33^{\circ}-53^{\circ}$ suggested by the deconvolved aspect ratio, agree well with the values obtained by Pinte et al. (2008) of, respectively, $-37^{\circ} \pm 5^{\circ}$ and $45^{\circ}-53^{\circ}$ from scattered light imaging.

A fit to the $1.3 \mathrm{~mm}$ visibilities done in Pinte et al. (2008) provides a rough disc mass estimate of $0.1 M_{\odot}$, with an uncertainty of a factor of few, dominated by the adopted dust emissivity and gas-to-dust mass ratio in the model.

\subsection{Molecular Lines}

Emission of ${ }^{12} \mathrm{CO}$ and ${ }^{13} \mathrm{CO} J=2-1$ was detected toward IM Lup, and an upper limit on $\mathrm{C}^{18} \mathrm{O} 2-1$ obtained. Figure 2 shows the zero moment (integrated emission, contours) and first moment (velocity centroid, colour scale) of the ${ }^{12} \mathrm{CO}$ and ${ }^{13} \mathrm{CO}$ emission from IM Lup. Significantly detected ${ }^{12} \mathrm{CO}$ emission extends to 5" from the star (roughly 900 AU for IM Lup). This is more than double the size inferred from the dust continuum image, and Sect. 4 discusses if this is due to different sensitivity in these two tracers or if the gas disc indeed extends further than the dust disc. The aspect ratio (5/3), suggesting an inclination of $53^{\circ} \pm 4^{\circ}$, and orientation $\mathrm{PA}=-36 \pm 5^{\circ}$ of the $\mathrm{CO}$ disc, agrees with the continuum image (Sect. 3.1) and scattered light imaging results (Pinte et al. 2008). 


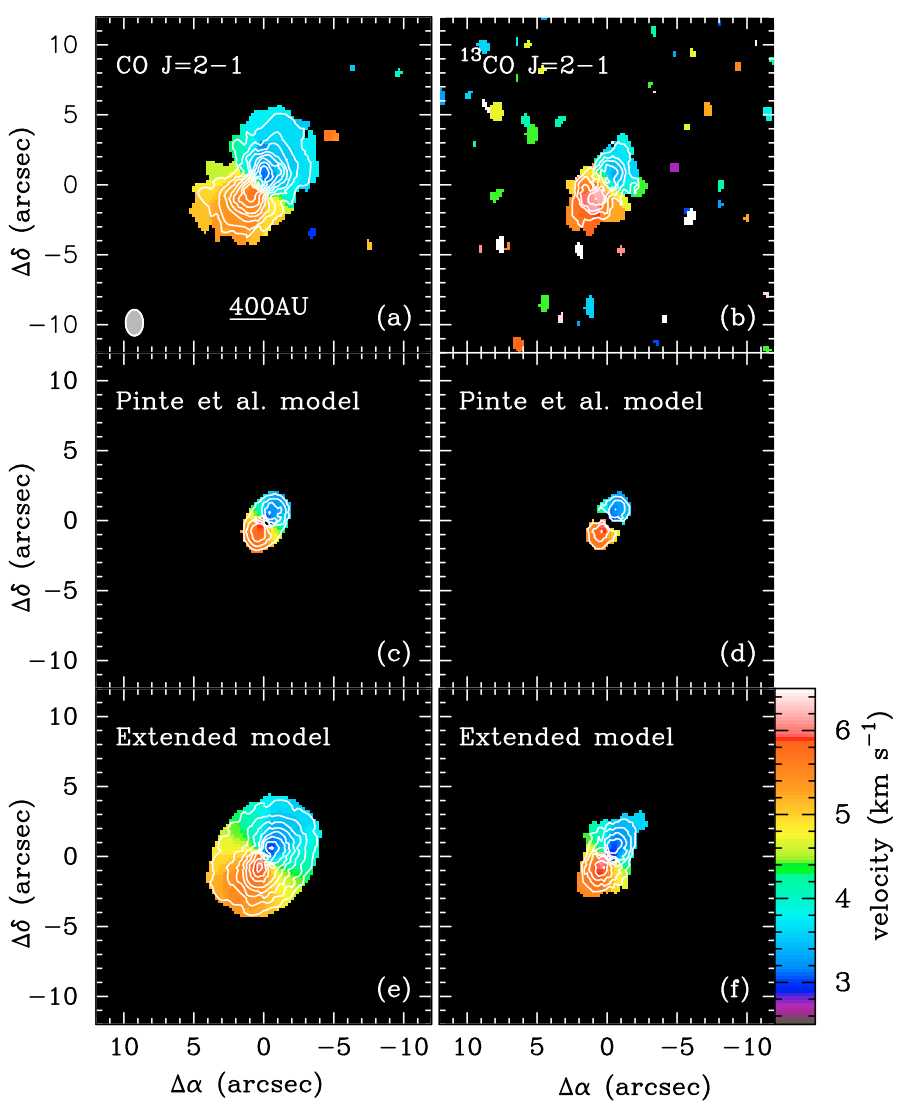

Fig. 2. a, b) First moment maps in the ${ }^{12} \mathrm{CO}$ and ${ }^{13} \mathrm{CO} J=2-1$ lines, from $1.9 \mathrm{~km} \mathrm{~s}^{-1}$ to $6.9 \mathrm{~km} \mathrm{~s}^{-1}$ observed towards IM Lup. These maps are created using the Miriad task "moment" with clip levels of 0.5 and $0.3 \mathrm{Jy}$ respectively. The integrated emission of ${ }^{12} \mathrm{CO} \mathrm{J}=2-1$ is shown in contours of $1,2,3, \ldots \times 500 \mathrm{mJy}$, and that of ${ }^{13} \mathrm{CO} J=2-1$ with 1 , $2,3, \ldots \times 160$ mJy contours. c, d) First moment and integrated emission maps calculated using Pinte et al. model and same clip level, velocity range and contour levels as in a) and b). e, f) First moment and integrated emission maps calculated using extended disc model (described in Sect. 4.2) with model parameters $\Sigma_{400}=2 \times 10^{21} \mathrm{~cm}^{-2}$ and $p=1$. The clip level, velocity range and contour levels are as in a), b), c) and d).

The first moment images of Fig. 2 show velocity patterns indicative of a rotating disc inclined with respect to the line of sight. This is also seen in Fig. 3, which presents the ${ }^{12} \mathrm{CO},{ }^{13} \mathrm{CO}$, and $\mathrm{C}^{18} \mathrm{O}$ spectra averaged over $8^{\prime \prime} \times 8^{\prime \prime}$ boxes around IM Lup. The ${ }^{12} \mathrm{CO}$ and ${ }^{13} \mathrm{CO}$ lines are double-peaked and centered on $v_{\mathrm{LSR}}=4.4 \pm 0.3 \mathrm{~km} \mathrm{~s}^{-1}$. Figures 4 and 5 show the channel maps of the ${ }^{12} \mathrm{CO}$ and ${ }^{13} \mathrm{CO}$ emission, respectively, revealing the same velocity pattern also seen from the first-moment maps and the spectra. The Keplerian nature of the velocity pattern is most clearly revealed by Fig. 6, which shows the position-velocity diagram of the ${ }^{12} \mathrm{CO}$ emission along the major axis of the disc. In Sect. 4, we derive a stellar mass of $1.2 M_{\odot}$, and, as an illustration, the rotation curves for stellar masses of $0.8,1.2$, and $1.6 M_{\odot}$ are plotted in Fig. 6.

Using single-dish ${ }^{12} \mathrm{CO} 3-2$ observations, van Kempen et al. (2007) first identified molecular gas directly associated with IM Lup, but they also conclude that the $v_{\mathrm{LSR}}$-range of 4 to $6 \mathrm{~km} \mathrm{~s}^{-1}$ is dominated by gas distributed over a larger $\left(>30^{\prime \prime}\right)$ scale. In our ${ }^{12} \mathrm{CO} 2-1$ data this same $v_{\mathrm{LSR}}$-range is also likely affected: where the single-dish ${ }^{12} \mathrm{CO} 3-2$ spectrum from van Kempen et al. shows excess emission over $v_{\mathrm{LSR}}=$ 4-6 $\mathrm{km} \mathrm{s}^{-1}$, the red peak of our ${ }^{12} \mathrm{CO} 2-1$ spectrum, which lies in this $v_{\mathrm{LSR}}$-range, is weaker than the blue peak at $+3.5 \mathrm{~km} \mathrm{~s}^{-1}$.

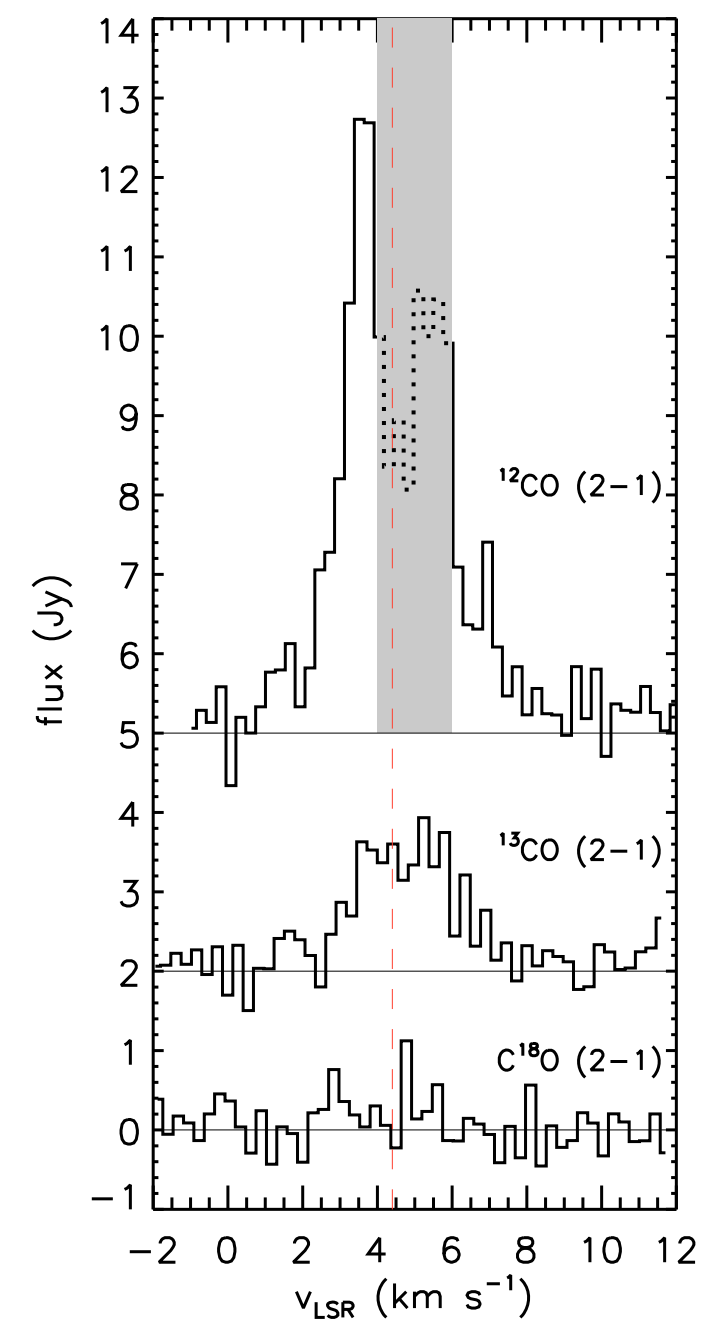

Fig. 3. ${ }^{12} \mathrm{CO},{ }^{13} \mathrm{CO}$, and $\mathrm{C}^{18} \mathrm{O} J=2-1$ line spectra summed over $8^{\prime \prime} \times 8^{\prime \prime}$ regions centered on the location of IM Lup. The ${ }^{13} \mathrm{CO}$ and ${ }^{12} \mathrm{CO}$ spectra are shifted upward by 2 and $5 \mathrm{Jy}$, respectively. The dashed red line shows the line centre at $v_{\mathrm{LSR}}=4.4 \mathrm{~km} \mathrm{~s}^{-1}$. The grey zone indicates the range from 4 to $6 \mathrm{~km} \mathrm{~s}^{-1}$ where the ${ }^{12} \mathrm{CO}$ line is significantly affected by the foreground absorption; the corresponding part of the ${ }^{12} \mathrm{CO}$ spectrum is plotted with a dotted line.

We suspect that absorption by the same foreground layer identified by van Kempen et al. is responsible for this decrement, while its emission is filtered out by the interferometer. The ${ }^{13} \mathrm{CO}$ $2-1$ spectrum is symmetric, suggesting that the foreground layer is optically thin in this line.

The spatial extent of the line emission is further explored in Fig. 7 which plots the ${ }^{12} \mathrm{CO}$ and ${ }^{13} \mathrm{CO} \mathrm{J}=2-1$ vector-averaged line fluxes against projected baseline length. The ${ }^{12} \mathrm{CO}$ flux is integrated from 2.5 to $4.0 \mathrm{kms}^{-1}$ to avoid the range where foreground absorption affects the line. The ${ }^{13} \mathrm{CO}$ flux does not suffer from absorption and is integrated over its full extent from 2.5 to $6.9 \mathrm{kms}^{-1}$. Comparing the curves of Fig. 7 to those of the continuum flux versus baseline lengths (Fig. 8) it is clear that the line flux is much more dominated by short spacings $(<40 \mathrm{k} \lambda)$. This profile may indicate the presence of a larger structural component (outer disc or envelope), combined with the disc emission (see Jørgensen et al. 2005, Fig. 2). We explore disc structure beyond $400 \mathrm{AU}$ in the following section. 

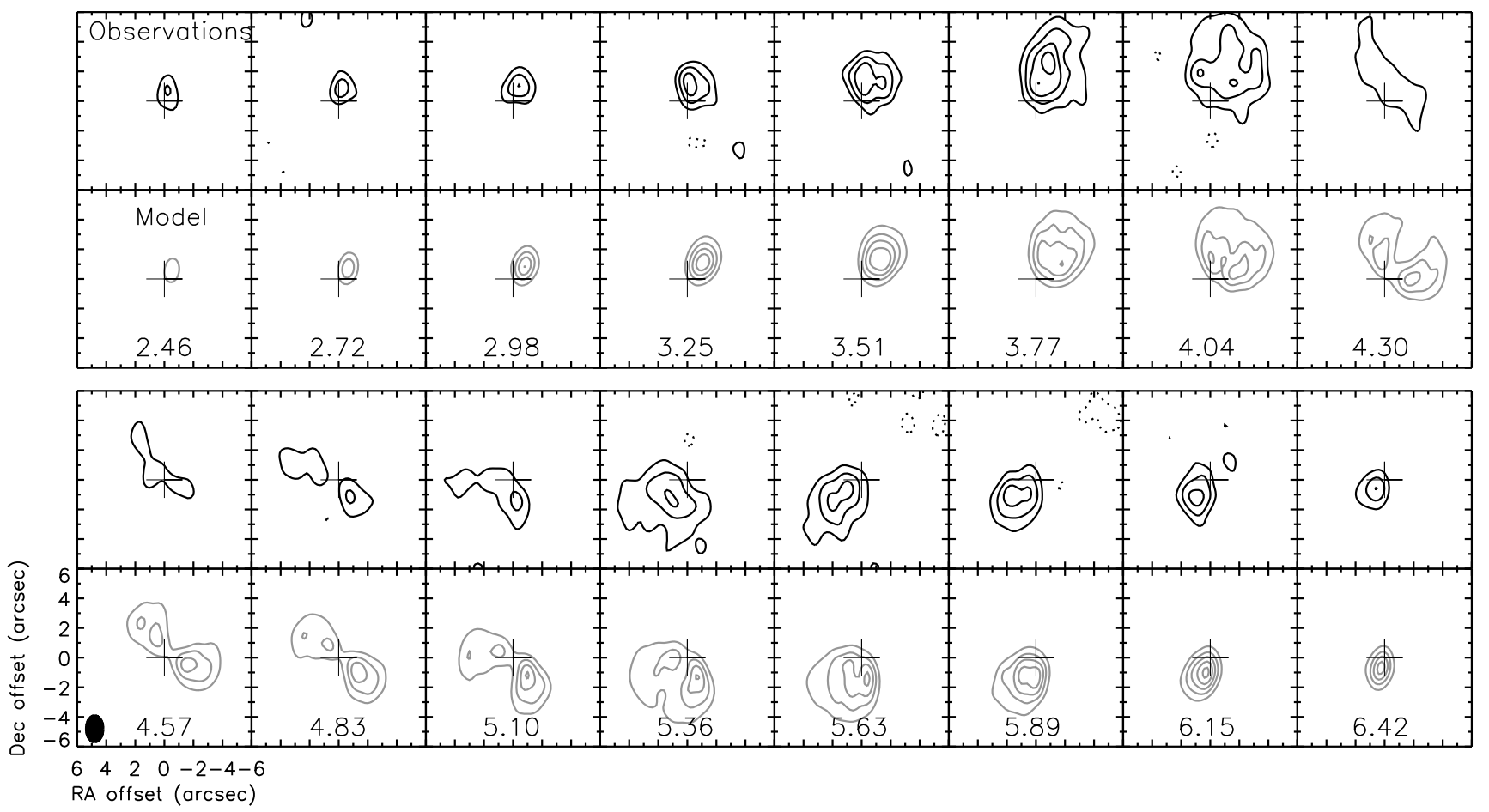

Fig. 4. The black contours show the observed ${ }^{12} \mathrm{CO} J=2-1$ emission in the velocity range from 2.46 to $6.42 \mathrm{~km} \mathrm{~s}^{-1}$. Alongside the observations, the panels with grey contours show the calculated emission from the extended disc model described in Sect. 4.2, with parameters $\Sigma_{400}=2 \times 10^{21} \mathrm{~cm}^{-2}$ and $p=1$. Labels indicate the velocity of each channel. The lower left corner of bottom-left panel shows the size and position angle of the synthesized beam. The contour levels are $-1,1,2,3,4 \times 400$ mJy beam $^{-1}(\sim 3 \sigma)$ in all panels.
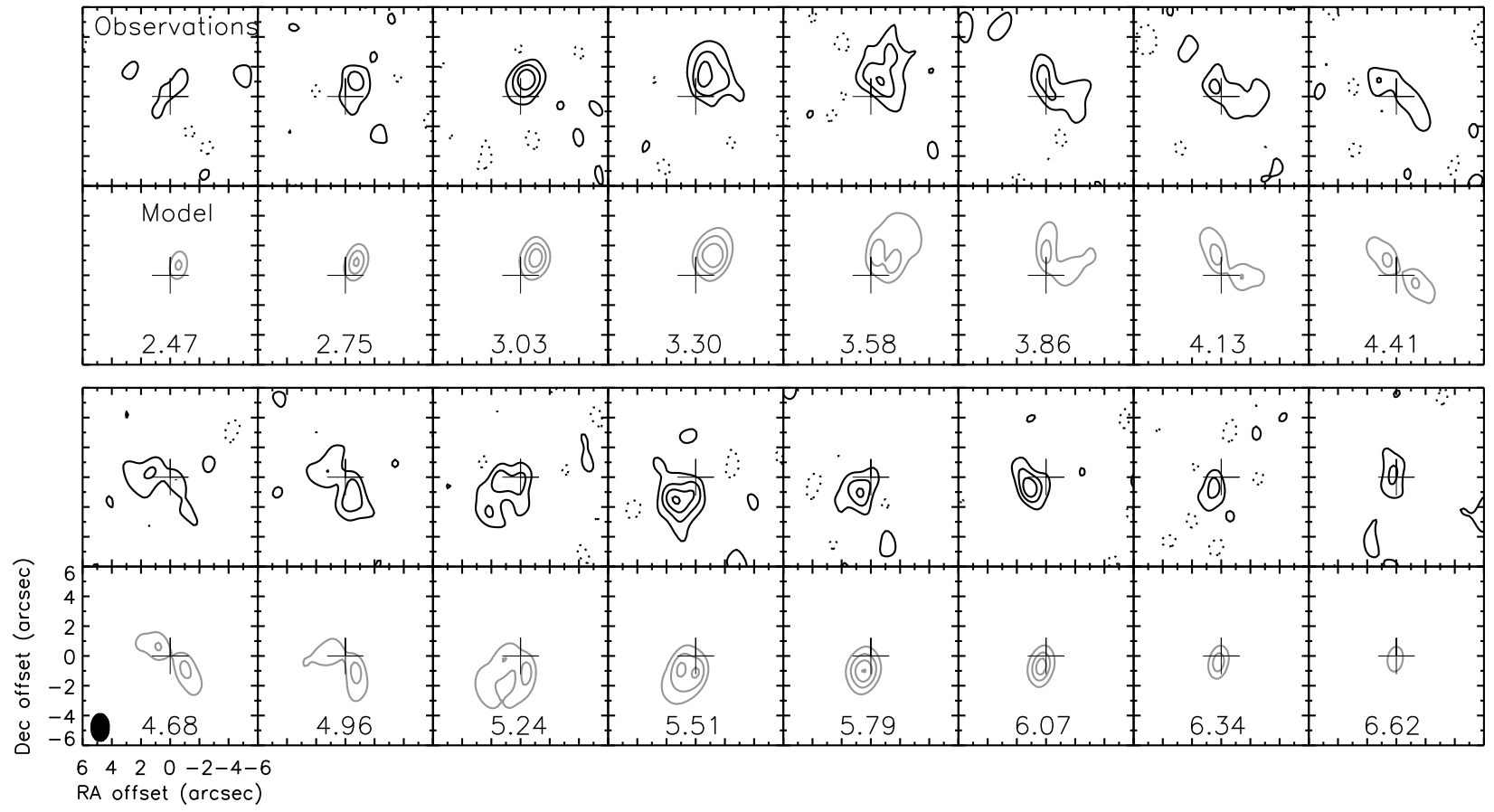

Fig. 5. Channel maps of the observed ${ }^{13} \mathrm{CO} J=2-1$ emission at the velocities where the line is detected are shown in black contours. For comparison, the line emission calculated from our extended disc model described in Sect. 4.2 is shown in grey contours. The model parameters are $\Sigma_{400}=2 \times 10^{21} \mathrm{~cm}^{-2}$ and $p=1$. Labels indicate the velocity of each channel. The lower left corner of bottom-left panel shows the size and position angle of the synthesized beam. The contour levels are $-1,1,2,3,4 \times 200 \mathrm{mJy} \mathrm{beam}^{-1}(\sim 2 \sigma)$ in all panels. 


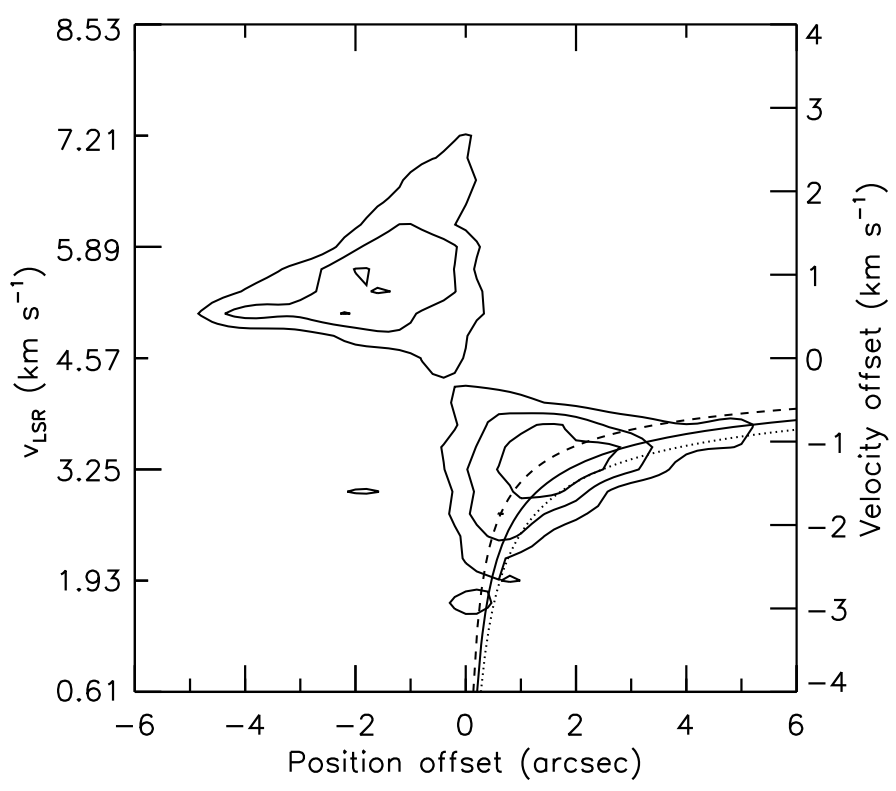

Fig. 6. Position-velocity diagram of the ${ }^{12} \mathrm{CO} 2-1$ line emission along the disc's major axis. Contour levels are $(1,2,3, \ldots) \times 400 \mathrm{mJy}(\sim 3 \sigma)$. For comparison, the thick solid line corresponds to Keplerian rotation around a $1.2 M_{\odot}$ star; dashed and dotted lines correspond to the stellar masses of 0.8 and of $1.6 M_{\odot}$, respectively.

\section{Discussion}

The results of the previous section show that IM Lup is surrounded by a gaseous disc in (roughly) Keplerian rotation. The gas disc has a radius of $900 \mathrm{AU}$, and its surface density may have a break around a radius of 400 AU. In contrast, the size of the dust disc is constrained to a radius of $400 \mathrm{AU}$ by our continuum data and the modelling of Pinte et al. (2008). This section explores if the gas and dust have the same spatial distribution (in which case different sensitivity levels need to explain the apparent difference in size) or if the gas and dust are differently distributed radially. First we investigate whether the model of Pinte et al. (2008) can explain the molecular line observations (Sect. 4.1). After we conclude that this is not the case, we construct new models for the gas disc (Sect. 4.2) describing their best-fit parameters, and compare them to the dust disc (Sect. 4.3).

\subsection{Molecular-line emission from the dust-disc model}

Recently, Pinte et al. (2008) present a detailed model for the disc around IM Lup based on the full SED, scattered light images at multiple wavelengths from the Hubble Space Telescope, near- and mid-infrared spectroscopy from the Spitzer Space Telescope, and continuum imaging at $1.3 \mathrm{~mm}$ with the Submillimeter Array.

Based on the two-dimensional density and temperature structure of the Pinte et al. model, with $M=0.1 M_{\odot}, R_{\text {out }}=$ $400 \mathrm{AU}$, and $i=50^{\circ}$, we calculate the resulting line intensity of the ${ }^{12} \mathrm{CO}$ and ${ }^{13} \mathrm{CO} J=2-1$ lines. To generate the input model for the molecular excitation calculations, we adopt a gas-to-dust mass ratio of 100 and molecular abundances typical for the dense interstellar medium (Frerking et al. 1982; Wilson \& Rood 1994): a ${ }^{12} \mathrm{CO}$ abundance with respect to $\mathrm{H}_{2}$ of $10^{-4}$ and a ${ }^{12} \mathrm{CO} /{ }^{13} \mathrm{CO}$ abundance ratio of 77 . No freeze-out or photodissociation of $\mathrm{CO}$ is included. The velocity of the material in the disc is described by Keplerian rotation around a $1.0 M_{\odot}$ star plus a Gaussian microturbulent velocity field with a FWHM of $0.16 \mathrm{~km} \mathrm{~s}^{-1}$; the exact value of the latter parameter has little effect on the results. Using the molecular excitation and radiative transfer code RATRAN (Hogerheijde \& van der Tak 2000) and $\mathrm{CO}-\mathrm{H}_{2}$ collision rates from the Leiden Atomic and Molecular Database (LAMBDA ${ }^{3}$, Schöier et al. 2005) we calculate the sky brightness distribution of the disc in the ${ }^{12} \mathrm{CO}$ and ${ }^{13} \mathrm{CO} J=2-1$ lines for its distance of $190 \mathrm{pc}$. From the resulting image cube, synthetic visibilities corresponding to the actual $(u, v)$ positions of our SMA data were produced using the MIRIAD package (Sault et al. 1995). Subsequent Fourier transforming, cleaning, and image restoration was performed with the same software.

Figure 2 compares the zeroth-moment (integrated intensity; contours) and first-moment (velocity-integrated intensity; colour scale) maps of the resulting synthetic images to the observations. Clearly, the Pinte et al. model produces ${ }^{12} \mathrm{CO}$ and ${ }^{13} \mathrm{CO}$ 2-1 emission with spatial extents and intensities too small by a factor close to two. In Fig. 7 it is clear that the Pinte et al. model fails to reproduce the ${ }^{12} \mathrm{CO}$ and ${ }^{13} \mathrm{CO}$ line fluxes at short projected baseline lengths, but is consistent with the observations longward of $40 \mathrm{k} \lambda$ that correspond to spatial scales $\leq 500 \mathrm{AU}$. Our comparison with Pinte et al. model thus suggests that the gas extends much further than $400 \mathrm{AU}$ from the star.

The observed $1.3 \mathrm{~mm}$ continuum emission traces the extent of larger dust particles (up to millimetre in size). Pinte et al. (2008) show that their 400 AU model reproduces these observations well. In Sect. 4.3 we explore to what level larger particles can be present outside $400 \mathrm{AU}$.

\subsection{Extending the gas disc beyond $400 \mathrm{AU}$}

As mentioned in Sect. 3.2, the CO line flux as function of projected baseline length suggests a possible break in the emission around $40 \mathrm{k} \lambda$ (Fig. 7). Results of Sect. 4.1 show that the Pinte et al. model, while providing a good description of line fluxes at small spatial scales (baselines $>40 \mathrm{k} \lambda$ ), requires a more extended component to match the observed line fluxes (baselines $<40 \mathrm{k} \lambda$ ). In this section we extend the Pinte et al. model by simple radial power laws for the gas surface density and temperature, and place limits on the gas column densities in the region between 400 and $900 \mathrm{AU}$.

Table 1 lists the model parameters. For radii smaller than $400 \mathrm{AU}$, the radial and vertical density distribution of the material follows the Pinte et al. model. As in Sect. 4.1 we adopt "standard" values of gas-to-dust mass ratio and molecular abundances, and a Gaussian microturbulent velocity field with equivalent line width of $0.16 \mathrm{~km} \mathrm{~s}^{-1}$. Unlike the calculations of Sect. 4.1 we add as free parameters the stellar mass $M_{\star}$ and the gas kinetic temperature. For the latter, we follow the twodimensional structure prescribed by Pinte et al., but scale the temperatures upward by a factor $f$ with $1 \leq f \leq 2$. This corresponds to a decoupling of the gas and dust temperatures, as may be expected at the significant height above the midplane where the ${ }^{12} \mathrm{CO}$ and ${ }^{13} \mathrm{CO}$ lines originate (see, e.g., Qi et al. 2006; Jonkheid et al. 2004). Because the highly red- and blue-shifted line emission (line wings) comes from regions closer to the star than $400 \mathrm{AU}$ and is optically thick, factor $\mathrm{f}$ is determined by the observed fluxes in the line wings. The molecular excitation and synthetic line data are produced in the same way as described in Sect. 4.1.

${ }^{3}$ http://www.strw.leidenuniv.nl/ moldata/ 

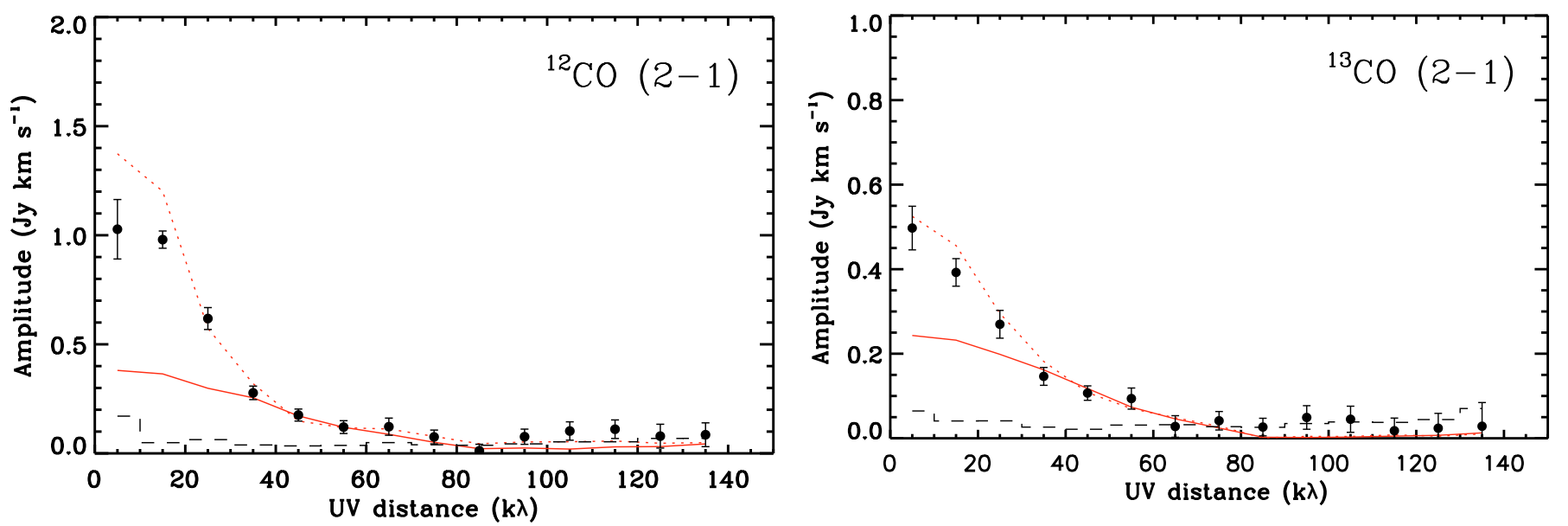

Fig. 7. The left and the right panel show vector-averaged ${ }^{12} \mathrm{CO}$ and ${ }^{13} \mathrm{CO}$ line flux (black symbols), respectively. Dashed black lines represent the zero-signal expectation value of our line visibility data. The calculated visibilities based on Pinte et al. model (full red line) and our extended disc model described in Sect. 4.2 (dotted red line) are shown for comparison. Our model parameters are $\Sigma_{400}=2 \times 10^{21} \mathrm{~cm}^{-2}$ and $p=1$. The ${ }^{12} \mathrm{CO}$ flux is integrated over the $0.8-4.0 \mathrm{~km} \mathrm{~s}^{-1}$ range and ${ }^{13} \mathrm{CO}$ over $2.5-6.9 \mathrm{~km} \mathrm{~s}^{-1}$, covering the full line width.

Outside $400 \mathrm{AU}$ we extend the disc to $900 \mathrm{AU}$, as suggested by the observed ${ }^{12} \mathrm{CO}$ image of Fig. 2, by simple radial power laws for the surface density and temperature, $\Sigma=$ $\Sigma_{400}(R / 400 \mathrm{AU})^{-p}$ and $T=T_{400}(R / 400 \mathrm{AU})^{-q}$. At $400 \mathrm{AU}$, the surface density is $\Sigma_{400}$ and the temperature is $T_{400}$; the parameter $p$ is assumed to be $\geq 0$. To limit the number of free parameters, we set $T_{400}=30 \mathrm{~K}$ and $q=0.5$; we assume that the disc is vertically isothermal and that the ${ }^{12} \mathrm{CO}$ abundance is $10^{-4}$, constant throughout the disc. At $R>400 \mathrm{AU}$, the disc thickness is set to $z_{\max }=100 \mathrm{AU}$ and the density $\rho(R, z)=\Sigma(R) / z_{\max }$ is vertically constant. For our free parameters $\Sigma_{400}$ and $p$, we assume that $\Sigma_{400} \leq 0.9 \mathrm{~g} \mathrm{~cm}^{-2}$ (vertical gas column density of $2 \times 10^{23} \mathrm{~cm}^{-2}$ ), the value at the outer radius of the Pinte et al. model. We have run a number of disc models, with the inner $400 \mathrm{AU}$ described by the Pinte et al. model (with the gas kinetic temperature scaled as described in the previous paragraph) and the region from 400 to $900 \mathrm{AU}$ described here by the disc extension. Figure 10 shows the surface density in the models that we have tested: within $400 \mathrm{AU}$ it is the surface density as in Pinte et al. (blue line) and between 400 and 900 AU different combinations of $\Sigma_{400}$ and $p$ (black lines). The models are tested against the observed ${ }^{12} \mathrm{CO}$ and ${ }^{13} \mathrm{CO} u v$-data, channel maps, spectra, and position-velocity plots. The comparison of modelled emission with $u v$-data for the line wings, $v_{\mathrm{LSR}}<3.0 \mathrm{~km} \mathrm{~s}^{-1}$ for ${ }^{12} \mathrm{CO}$ and $v_{\mathrm{LSR}}<3.5 \mathrm{~km} \mathrm{~s}^{-1}+v_{\mathrm{LSR}}>5.5 \mathrm{~km} \mathrm{~s}^{-1}$ for ${ }^{13} \mathrm{CO}$ is also examined.

Figure 10 shows the models that overproduce the observed emission with dashed black lines and those that underproduce it with dotted black lines. The full black lines correspond to the models that reproduce well our ${ }^{12} \mathrm{CO}$ and ${ }^{13} \mathrm{CO}$ data. The general area (beyond $400 \mathrm{AU}$ ) allowed by the models is shaded in Fig. 10 for guidance. It can be seen that the ${ }^{12} \mathrm{CO}$ and ${ }^{13} \mathrm{CO}$ observations constrain the column density of ${ }^{12} \mathrm{CO}$ at $R=900 \mathrm{AU}$ to $N_{\mathrm{CO}}=(0.05-1.0) \times 10^{18} \mathrm{~cm}^{-2}$, where the lower bound follows from the requirement that the ${ }^{12} \mathrm{CO}$ emission is sufficently extended and the upper bound from the requirement that the ${ }^{12} \mathrm{CO}$ and ${ }^{13} \mathrm{CO}$ peak intensity, and the extent of the ${ }^{13} \mathrm{CO}$ emission are not overestimated. The corresponding surface density at $900 \mathrm{AU}$ is $\Sigma_{900}=(0.2-4.0) \times 10^{-2} \mathrm{~g} \mathrm{~cm}^{-2}$, i.e., a vertical gas column density $(0.05-1.0) \times 10^{22} \mathrm{~cm}^{-2}$. Our data do not constrain the parameters $\Sigma_{400}$ and $p$, that determine how the surface density decreases from its value at the outer edge of the Pinte et al. model,

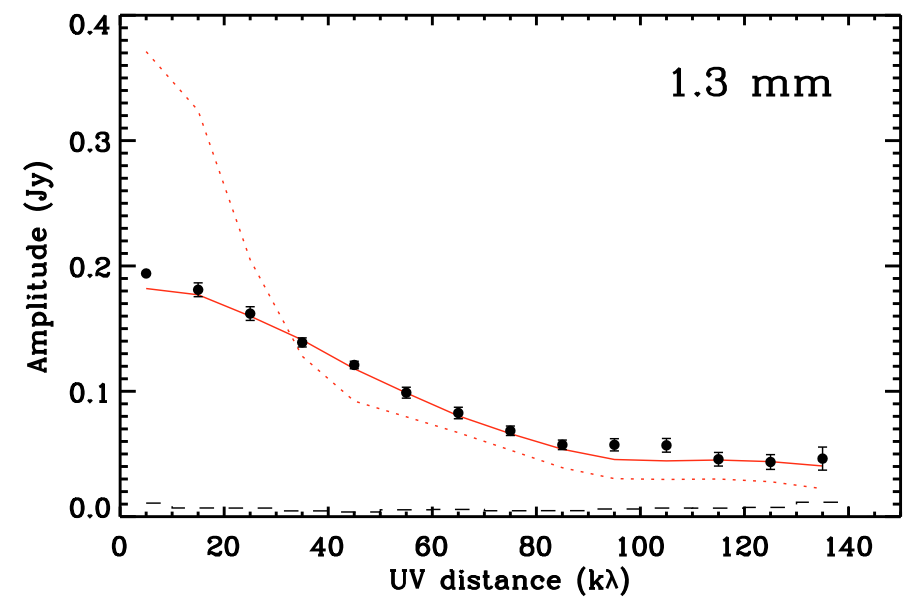

Fig. 8. Vector-averaged continuum flux as a function of projected baseline length (black symbols). Error bars show the variance within each annular bin. The dashed histogram shows the zero-signal expectation value. The full red line shows the continuum flux calculated from Pinte et al. model. The dotted red line corresponds to the extended disc model with $\Sigma_{400}=2 \times 10^{21} \mathrm{~cm}^{-2}$ and $p=1$ (see Sect. 4.2 for model description).

to its value at $900 \mathrm{AU}$. This is either a marked change from the power-law slope of $p=1$ found inside $400 \mathrm{AU}$ to $p=5$ beyond $400 \mathrm{AU}$, or a discontinuous drop by a factor $\sim 10-100$ in surface density at $400 \mathrm{AU}$.

Figure 7 compares observations to synthetic ${ }^{12} \mathrm{CO}$ and ${ }^{13} \mathrm{CO}$ line visibilities for our model with $\Sigma_{400}=2 \times 10^{21} \mathrm{~cm}^{-2}$ and $p=1$, plotted with dotted red lines. There is a good match between the model and the data for both transitions. In particular, the model reproduces well the change in the slope of visibilities, mentioned in Sect. 3.2. The match between the model (red lines) and observations is also seen in the line spectra, Fig. 9. Figures 4 and 5 show, respectively, the ${ }^{12} \mathrm{CO}$ and ${ }^{13} \mathrm{CO}$ channel maps (black contours) compared to our extended disc model (grey contours). It can therefore be seen that our model provides a good description not only of the line intensity at each channel (spectra), but also a very close match in the spatial extent of the emission in each spectral channel. 
Table 1. Model parameters.

\begin{tabular}{c|c|c}
\hline \hline Parameter & $R<400 \mathrm{AU}$ & $400 \leq R \leq 800 \mathrm{AU}$ \\
\hline Surface density & $\sum \propto R^{-1}$, see Pinte et al. (2008) & $\Sigma_{400}(R / 400 \mathrm{AU})^{-p}$ with $\Sigma_{400} \leq 0.9 \mathrm{~g} \mathrm{~cm}^{-2}, p \geq 0$ \\
Gas-to-dust mass ratio & 100 & 100 \\
Gas temperature structure & $f T_{\text {dust }}$ with $1 \leq f \leq 2$ & $T_{400}(R / 400 \mathrm{AU})^{-q}, T_{400}=30 \mathrm{~K}, q=0.5$ \\
Vertical structure & see Pinte et al. $(2008)$ & $T(z)=$ const., $\rho(z)=$ const.,,$z_{\max }=100 \mathrm{AU}$ \\
{$[\mathrm{CO}] /\left[\mathrm{H}_{2}\right]$} & $10^{-4}$ & $10^{-4}$ \\
{$\left[{ }^{12} \mathrm{CO}\right] /\left[{ }^{13} \mathrm{CO}\right]$} & 77 & 77 \\
$M_{\star}$ & $1.2 M_{\odot}$ & $1.2 M_{\odot}$ \\
Inclination & $50^{\circ}$ & $50^{\circ}$ \\
FWHM microtubulence & $0.16 \mathrm{~km} \mathrm{~s}^{-1}$ & $0.16 \mathrm{~km} \mathrm{~s}^{-1}$ \\
\hline
\end{tabular}

A good fit to the wings of the ${ }^{12} \mathrm{CO}$ and ${ }^{13} \mathrm{CO}$ spectra (Fig. 9, red lines) and the spatial distribution of the respective line fluxes at highly blue- and red-shifted velocities (lower panels, Figs. 4 and 5) is found for temperature scalings $f$ of 1.7 for ${ }^{12} \mathrm{CO}$ and 1.4 for ${ }^{13} \mathrm{CO}$. These values of $f$ suggest that the gas is somewhat warmer than the dust at the heights above the disc where the ${ }^{12} \mathrm{CO}$ and ${ }^{13} \mathrm{CO}$ emission originates, and more so at the larger height probed by the ${ }^{12} \mathrm{CO}$ line compared to the ${ }^{13} \mathrm{CO}$ line.

At the adopted disc inclination of $50^{\circ}$, the line peak separation provides a reliable constraint on the stellar mass. We find a best-fit of $M_{\star}=1.2 \pm 0.4 M_{\odot}$, where the uncertainty is dominated by our limited spectral resolution. This value is consistent with the rough estimate of $1 M_{\odot}$ from Pinte et al. (2008), but a few times higher than derived by Hughes et al. (1994).

We conclude that the surface density traced through ${ }^{12} \mathrm{CO}$ and ${ }^{13} \mathrm{CO}$ has a discontinuity around $R=400 \mathrm{AU}$ either in $\Sigma_{\mathrm{CO}}(R)$ or in its derivative $\mathrm{d} \Sigma_{\mathrm{CO}} / \mathrm{d} R$, or both. This may, or may not be an indication of an overall discontinuity of the gas surface density. A break in the temperature $T(R)$ cannot explain the observations, since our model already adopts a low temperature at the margin of ${ }^{12} \mathrm{CO}$ freeze-out in the outer regions. An alternative explanation for the observations is a radical drop in the abundance of $\mathrm{CO}$ (with respect to $\mathrm{H}_{2}$ and $\mathrm{H}$ ) or its radial derivative. Freeze-out onto dust grains or photodissociation can significantly reduce the gas-phase abundance of CO. In the next section we explore the limits that the dust emission can give us on the gas content outside $400 \mathrm{AU}$, and compare them to the ${ }^{12} \mathrm{CO}$ results.

\subsection{Comparing gas and dust at radii beyond $400 \mathrm{AU}$}

The previous section concluded that both the gas and the dust out to $400 \mathrm{AU}$ in the disc around IM Lup is well described by the model of Pinte et al., with the exception of gas temperatures that exceed the dust temperature at some height above the disc midplane. It also found that the gas disc needs to be extended to an outer radius of $900 \mathrm{AU}$, albeit with a significant decrease in the surface density of $\mathrm{CO}, \Sigma_{\mathrm{CO}}$, or in its first derivative, $\mathrm{d} \Sigma_{\mathrm{CO}} / \mathrm{d} R$ close to $400 \mathrm{AU}$.

Pinte et al. (2008) show that some dust is present outside $400 \mathrm{AU}$ as well, visible as an extended nebulosity in their $0.8 \mu \mathrm{m}$ scattered light images. At the same time, the visible lower scattering disc surface places a stringent limit on the surface density $\Sigma_{\text {dust }}$ of small dust particles outside 400 AU. Requiring the optical depth $\tau=\Sigma_{\text {dust }} \kappa<1$ and adopting an emissivity per gram of dust of $\kappa=(8-10) \times 10^{3} \mathrm{~cm}^{2} \mathrm{~g}^{-1}$ at $0.8 \mu \mathrm{m}$ (see first row of Table 1, Ossenkopf \& Henning 1994), we find $\Sigma_{\text {dust }} \leq$ $(1.0-1.3) \times 10^{-4} \mathrm{~g} \mathrm{~cm}^{-2}$. If we adopt the gas-to-dust mass ratio of 100 , this corresponds to $N_{\mathrm{H}_{2}} \leq(2.5-3.1) \times 10^{21} \mathrm{~cm}^{-2}$. Our

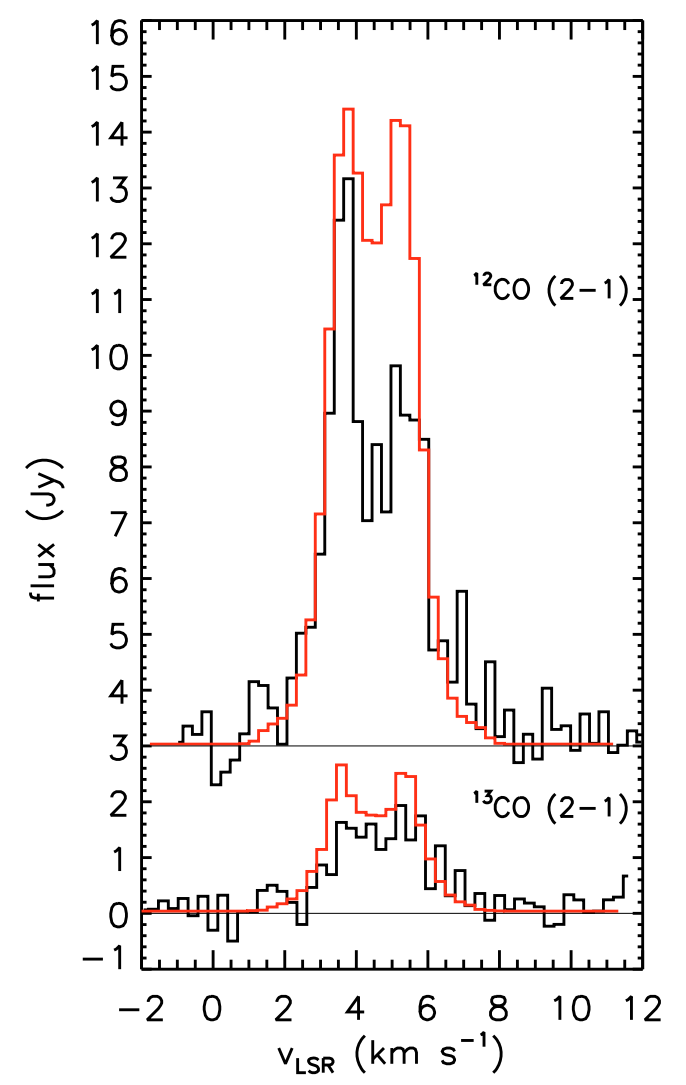

Fig. 9. ${ }^{12} \mathrm{CO}$ and ${ }^{13} \mathrm{CO} J=2-1$ line spectra averaged over a $8{ }^{\prime \prime} 0 \times 88^{\prime \prime} 0$ region centered on IM Lup. The ${ }^{12} \mathrm{CO}$ spectrum is shifted up by $3 \mathrm{Jy}$ for clarity. The red lines show the emission predicted from the extended disc model described in Sect. 4.2 with $\Sigma_{400}=2 \times 10^{21} \mathrm{~cm}^{-2}$ and $p=1$.

limit differs from that given in Pinte et al. (2008) $\left(0.2 \mathrm{~g} \mathrm{~cm}^{-2}\right)$ because we use dust opacities representative of small dust, while they assume considerable grain growth in disc midplane and thus use much lower dust opacities at $0.8 \mu \mathrm{m}$. The limit on surface density we derive is two orders of magnitude lower than the column density at the outer radius of $400 \mathrm{AU}$ of the Pinte et al. model. This indicates that either the dust surface density drops sharply at $400 \mathrm{AU}$, or that efficient grain growth beyond 400 AU has caused a significant decrease in dust near-IR opacity. As can be seen in Fig. 10, the upper limit on surface density of $(2.5-3.1) \times 10^{21} \mathrm{~cm}^{-2}$ is consistent with the gas surface density range inferred in Sect. 4.2 from our CO data, using the canonical $\mathrm{CO} / \mathrm{H}_{2}$ abundance of $10^{-4}$.

While $0.8 \mu \mathrm{m}$ imaging traces the small dust, our observations of $1.3 \mathrm{~mm}$ dust continuum emission, on the other hand, trace the millimetre-sized dust particles. In Fig. 8 we can see that the 


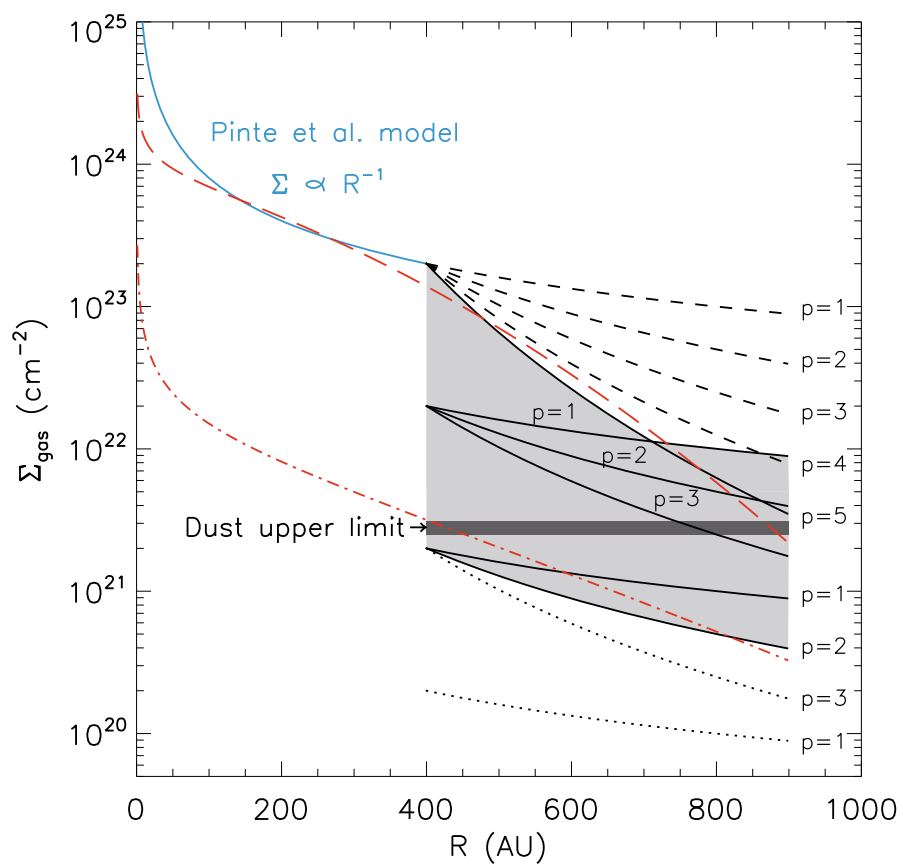

Fig. 10. Gas surface density in our disc models is plotted as a function of radius. Within $400 \mathrm{AU}$, it is identical to the Pinte et al. model shown with the full blue line. Outside $400 \mathrm{AU}$, we explore different power-law distributions, each plotted in black and marked with the corresponding slope $p$. The models which overestimate the observed ${ }^{12} \mathrm{CO}$ emission are plotted with dashed lines, while those that underpredict it are shown in dotted lines. The full black lines represent the models that fit well the ${ }^{12} \mathrm{CO} J=2-1$ emission, and define the shaded region which shows our constraint on $\Sigma_{\mathrm{CO}} /[\mathrm{CO}]$ in the outer disc, where a $\mathrm{CO}$ abundance of $[\mathrm{CO}]=10^{-4}$ is used. The upper limit on $\Sigma_{\text {dust }} g /$ d placed by scattered light images is shown with a dark grey line, with a gas-to-dust mass ratio $g / d=100$. For comparison, the red lines correspond to disc models with an exponential drop off as described in Hughes et al. (2008). The model shown with the long-dashed red line has parameters $\gamma=0.3, c_{1}=340 \mathrm{AU}$ and $c_{2}=3.1 \times 10^{24} \mathrm{~cm}^{-2}$ and fits the gas constraints. The model with the dash-dotted red line $\gamma=0.6, c_{1}=340 \mathrm{AU}$ and $c_{2}=1.8 \times 10^{23} \mathrm{~cm}^{-2}$ fits the scattered light constraints. No single model with a tapered outer edge can fit both these constraints and the constraints within $400 \mathrm{AU}$ simultaneously.

Pinte et al. model (full red line), with the radius of $400 \mathrm{AU}$, compares well to the observed continuum flux at all projected baseline lengths. On the other hand, the comparison of the $1.3 \mathrm{~mm}$ visibilities to our extended disc model with $\Sigma_{400}=2 \times 10^{21} \mathrm{~cm}^{-2}$ and $p=1$ shows that the model overestimates emission at short $u v$-distances (large spatial scales). A constant dust emissivity of $2.0 \mathrm{~cm}^{2} \mathrm{~g}^{-1}$ (emissivity of mm-sized grains, as in Draine 2006) was used throughout the disc in the calculation of $1.3 \mathrm{~mm}$ fluxes. Our model indicates that any dust present in the outer disc regions must be poor in mm-sized grains, i.e., have low millimetre wavelength opacities, while dust within 400 AU has likely undergone grain growth as found by Pinte et al. (2008). This further supports our choice of $\kappa$ at $0.8 \mu \mathrm{m}$ when estimating the upper limit on dust column (see above). Therefore, a viable model for the disc of IM Lup consists of an 'inner' disc extending to $400 \mathrm{AU}$ as described in Pinte et al. augmented with an 'outer disc' extending from 400 to 900 AU with a significantly reduced surface density (with negligible mass) but standard gas-to-dust mass ratio and $\mathrm{CO}-$ to- $\mathrm{H}_{2}$ ratios. The $\mathrm{SED}$ of this new model should not differ significantly to that of Pinte et al. model, and is therefore expected to provide a good match to the observed SED of IM Lup.

Hughes et al. (2008) find that the apparent difference between the extent of submillimetre dust and gas emission in several circumstellar discs can be explained by an exponential dropoff of surface density which naturally arises at the outer edge of a viscous disc. In Fig. 10 we show how, with a careful choice of parameters $\left(\gamma=0.3, c_{1}=340 \mathrm{AU}\right.$ and $\left.c_{2}=3.1 \times 10^{24} \mathrm{~cm}^{-2}\right)$, the model of Hughes et al. (2008) (red long-dashed line) can reproduce the surface density distribution of the models which describe well the ${ }^{12} \mathrm{CO} 2-1$ line emission. This model, and the one discussed below, are only examples. A proper modelling of IM Lup in the context of viscous disc models would require a revision of the entire disc structure both in terms of temperature and density, which is outside of the scope of the current work. We notice that the Hughes et al. models cannot simultaneously comply with the gas and dust constraints in the outer disc and the surface density derived by Pinte et al. (2008) in the inner disc. This is illustrated by the Hughes et al. (2008) model with parameters $\gamma=0.6, c_{1}=340 \mathrm{AU}$ and $c_{2}=1.8 \times 10^{23} \mathrm{~cm}^{-2}$, shown with the red dash-dotted line in Fig. 10. The surface density of this model outside $400 \mathrm{AU}$ is in agreement with observational constraints from gas and dust, but it is roughly two orders of magnitude lower than suface density from Pinte et al. (2008) within $400 \mathrm{AU}$.

In the standard theory of viscous discs (see Pringle 1981), irrespective of the initial density distribution, a radially smooth surface density distribution with a tapered outer edge is rapidly reached. If there is a significant change in the nature of the viscosity inside and outside of $400 \mathrm{AU}$, discontinuities in the equilibrium surface density may follow. Such changes could, for example, result from differences in the ionization structure of the disc or from a drop of the surface density below some critical level. Here we explore some scenarios that could explain this:

A young disc. An extreme example of such a configuration is a disc where the inner $400 \mathrm{AU}$ follows the standard picture of a viscous accretion disc, but where the region outside $400 \mathrm{AU}$ has not (yet) interacted viscously with the inner disc. This outer region may be the remnant of the flattened, rotating prestellar core that has not yet made it onto the viscous inner disc. This configuration, reminiscent of the material around the object L1489 IRS (Brinch et al. 2007), suggests that IM Lup would only recently have emerged from the embedded phase. L1489 IRS showed clear inward motion in its rotating envelope. Our observations limit any radial motions in the gas between 400 and $900 \mathrm{AU}$ to $<0.2 \mathrm{~km} \mathrm{~s}^{-1}$, or $20 \%$ of the Keplerian orbital velocities at these radii. Furthermore, for the $900 \mathrm{AU}$ structure to survive for the lifetime of IM Lup of 0.1-0.6 Myr, inward motions cannot exceed $\sim 10^{-2} \mathrm{~km} \mathrm{~s}^{-1}$. Any mass inflow is therefore small, and the material between 400 and 900 AU is likely on Keplerian orbits.

A companion body. Another explanation for the break in the disc density structure around $400 \mathrm{AU}$ would be the presence of a companion near this radius. A companion of $\sim 1 M_{\text {Jup }}$ at 400 AU could open a gap in the disc and affect the viscous disc spreading. No companions at this separation are visible in the HST images of (Pinte et al. 2008) or in $K$-band direct imaging (Ghez et al. 1997). Whether these observations exclude this scenario is unclear: it requires modelling of the orbital evolution of a companion in a viscously spreading disc and calculation of the observational mass limits at the age of IM Lup. This is beyond the scope of this paper.

Gas to dust ratio. While our model is consistent with standard gas-to-dust and $\mathrm{CO}-\mathrm{to}_{\mathrm{H}} \mathrm{H}_{2}$ ratios beyond $400 \mathrm{AU}$, this is not the only solution. Instead of adopting these standard 
ratios, which requires explaining the drop in $\Sigma$ or $\mathrm{d} \Sigma / \mathrm{d} R$ around $400 \mathrm{AU}$, we can hypothesize that the gas $\left(\mathrm{H}_{2}\right.$ or $\left.\mathrm{H}\right)$ surface density is continuous out to $900 \mathrm{AU}$ and that both the CO-to- $\mathrm{H}_{2}$ and dust-to-gas ratios show a break around $400 \mathrm{AU}$. This scenario requires a drop between 400 and $900 \mathrm{AU}$ of the $\mathrm{CO}$ abundance by a factor between 10 and 200, and of the dust-to-gas mass ratio by a factor $\geq 90$. These drops can be sudden, with a discontinuity at $400 \mathrm{AU}$, or more gradual, with a rapid decline of the two ratios from 400 to 900 AU. Since a low amount of dust emission outside $400 \mathrm{AU}$ is observed both at wavelengths of $\sim 1 \mathrm{~mm}$ (our data) and $\sim 1 \mu \mathrm{m}$ (Pinte et al. 2008), the overall dust-to-gas ratio is likely affected, and not just the individual populations of small and large grains.

Dust radial drift and photoevaporation. If a large fraction of the dust is removed from the disc regions outside $400 \mathrm{AU}$, the increased penetration of ultraviolet radiation could explain the drop in ${ }^{12} \mathrm{CO}$ surface density through increased photodissociation (van Zadelhoff et al. 2003). Radial drift of dust particles due to the gas drag force (Whipple 1972; Weidenschilling 1977) is a possible scenario in circumstellar discs. The difference in velocity between the dust, in Keplerian rotation, and gas, subKeplerian because of the radial pressure gradient, can cause dust particles to lose angular momentum and drift inward. The optimal drift particle size depends on the gas density, Keplerian rotation frequency and hydrostatic sound speed. Most dust evolution models focus on the inner $100 \mathrm{AU}$ of discs, relevant to planet formation. In these regions, the grains from $100 \mu \mathrm{m}$ to about $0.1 \mathrm{~m}$ efficiently migrate inwards on a timescale shorter than 2 Myr. However, the optimal grain size for inward drift decreases with the gas density. Our modelling of the disc region from $400 \mathrm{AU}$ to $900 \mathrm{AU}$, predicts surface densities of $\sim 10^{-3} \mathrm{~g} \mathrm{~cm}^{-2}$, low enough even for sub-micron-sized particles to drift inward (to $<400 \mathrm{AU}$ ). For the estimated age of IM Lup of $0.1-0.6 \mathrm{Myr}$, all particles larger than $0.1-0.02 \mu \mathrm{m}$ will have migrated inward. This process leaves the outer disc unshielded by dust against UV radiation. Infrared emission of PAHs may be used to trace the disc surface in this scenario. However, Geers et al. (2007) do not detect PAH emission at $3.3 \mu \mathrm{m}$ in their VLT-ISAAC L-band observations of IM Lup. This may indicate that either there are not enough PAHs in the disc or that they are not exposed to a significant level of UV flux. The latter possibility allows the outer disc to remain molecular. Otherwise, the outer disc is exposed to photodissociating radiation, destroying much of the $\mathrm{CO}$ and likely also a significant fraction of the $\mathrm{H}_{2}$ given the limit on the dust surface density of $10^{21} \mathrm{~cm}^{-2}$ corresponding to $A_{\mathrm{V}} \approx 1^{\mathrm{mag}}$. In this scenario, the outer disc between 400 and 900 AU would be largely atomic and possibly detectable through $21 \mathrm{~cm}$ observations of $\mathrm{H} \mathrm{I}$, or line observations of C I at 609 and $370 \mu \mathrm{m}$ or C II at $158 \mu \mathrm{m}$. If photoevaporation is efficient in this region it may remove the (atomic) gas and reduce the gas surface density further. Therefore, a combined effect of efficient drift, photodissociation and photoevaporation in the outermost disc regions may be a reason for the low gas and dust density observed. The efficiency of these processes decreases with density and perhaps the density at $400 \mathrm{AU}$ is high enough so that material is no longer efficiently removed from the disc. Only the detailed simultaneous modelling of drift, photodissociation and photoevaporation could test this scenario.

\section{Conclusions}

We probe the kinematics and the distribution of the gas and dust in the disc around IM Lup through molecular gas and continuum dust emission. Our SMA observations resolve the disc structure down to scales of $200 \mathrm{AU}$, and allow us to probe the structure of the inner disc (<400 AU) and the outer disc (400-900 AU). Our main conclusions can be summarized as follows.

- The ${ }^{12} \mathrm{CO}$ and ${ }^{13} \mathrm{CO}$ emission extends to $900 \mathrm{AU}$ from IM Lup, much further than the outer radius of 400 AU inferred earlier from dust measurements.

- The $\mathrm{H}_{2}$ gas surface density in the region between 400 and $900 \mathrm{AU}$ lies in the range of $5 \times 10^{20}$ to $10^{22} \mathrm{~cm}^{-2}$, using the standard CO-to- $\mathrm{H}_{2}$ ratio of $10^{-4}$.

- The disc is in Keplerian rotation around a central mass of $1.2 \pm 0.4 M_{\odot}$. Infall motions, if present in the outer disc, are negligible at $<0.2 \mathrm{~km} \mathrm{~s}^{-1}$.

- The molecular line emission from the inner disc, within $400 \mathrm{AU}$, is well described by the model of Pinte et al. (2008), except that the gas temperature in the layers dominating the line emission of ${ }^{12} \mathrm{CO}$ and ${ }^{13} \mathrm{CO}$ exceeds the dust temperature by factors 1.7 and 1.4 , respectively.

- Outside 400 AU, the surface densities of the molecular gas, as traced through ${ }^{12} \mathrm{CO}$ and ${ }^{13} \mathrm{CO}$, of small $(\sim 1 \mu \mathrm{m})$ dust grains, and of larger $(\sim 1 \mathrm{~mm})$ dust grains have a break in their radial dependence. At $400 \mathrm{AU}$, the dust surface density (in small grains) drops by a factor $\sim 100$, while the gas surface density shows a comparable drop of a factor 10-200 or steepens its radial power-law slope from $p=1$ to $5 \leq p \leq 8$.

Our observations show that the disc around IM Lup consists of two regions. The inner $400 \mathrm{AU}$ is well described by a 'standard' accretion disc; the region between 400 and $900 \mathrm{AU}$ has a much lower surface density as traced through dust grains with sizes from $\sim 1 \mu \mathrm{m}$ to $1 \mathrm{~mm}$ and through CO emission. Our observations do not tell us if this outer region consists of material from the original prestellar core that has not (yet) made it onto the viscous accretion disc, or of material that is part of the disc but has had a different evolution. Sensitive, spatially resolved observations at various (sub) millimetre wavelengths, as may be obtained with the Atacama Large Millimeter Array may help to assess whether significantly different grain populations exist inside and outside of $400 \mathrm{AU}$. With the same telescope, very high signal-to-noise observations of ${ }^{12} \mathrm{CO}$ lines at high spectral resolution may allow determination of any radial (inward or outward) motions in the $>400$ AU gas. Spatially resolved midinfrared imaging in several emission bands of PAHs, as could be obtained with the VISIR instrument on VLT, would shed light on the question if the 400-900 AU zone in the disc is largely photodissociated or -ionised. Detailed modelling of dust evolution in the outer disc may answer whether radial drift is responsible for the low column of dust beyond 400 AU in IM Lup disc.

Acknowledgements. The research of O.P. and M.R.H. is supported through a VIDI grant from the Netherlands Organisation for Scientific Research. We would like to thank our Leiden colleagues Anders Johansen and Richard D. Alexander for valuable insights and discussions, as well as C. P. Dullemond, A. Juhász and others at the Star and Planet Formation Department of MPIA Heidelberg for their help and advice during the stay of O.P. in March 2008. Finally, we are grateful to E. F. van Dishoeck for her support and guidance throughout the writing of this paper.

\section{References}

Baraffe, I., Chabrier, G., Allard, F., \& Hauschildt, P. 1998, VizieR Online Data Catalog, 333, 70403

Brinch, C., Crapsi, A., Hogerheijde, M. R., \& Jørgensen, J. K. 2007, A\&A, 461, 1037

Chiang, E. I., \& Goldreich, P. 1997, ApJ, 490, 368

D'Alessio, P., Merín, B., Calvet, N., Hartmann, L., \& Montesinos, B. 2005, Rev. Mex. Astron. Astrofis., 41, 61 
D’Antona, F., \& Mazzitelli, I. 1994, ApJS, 90, 467

Dartois, E., Dutrey, A., \& Guilloteau, S. 2003, A\&A, 399, 773

Draine, B. T. 2006, ApJ, 636, 1114

Dullemond, C. P., Dominik, C., \& Natta, A. 2001, ApJ, 560, 957

Frerking, M. A., Langer, W. D., \& Wilson, R. W. 1982, ApJ, 262, 590

Geers, V. C., van Dishoeck, E. F., Visser, R., et al. 2007, A\&A, 476, 279

Ghez, A. M., McCarthy, D. W., Patience, J. L., \& Beck, T. L. 1997, ApJ, 481, 378

Guilloteau, S., \& Dutrey, A. 1998, A\&A, 339, 467

Hogerheijde, M. R., \& van der Tak, F. F. S. 2000, A\&A, 362, 697

Hughes, A. M., Wilner, D. J., Qi, C., \& Hogerheijde, M. R. 2008, ApJ, 678, 1119

Hughes, J., Hartigan, P., Krautter, J., \& Kelemen, J. 1994, AJ, 108, 1071

Isella, A., Testi, L., Natta, A., et al. 2007, A\&A, 469, 213

Jonkheid, B., Faas, F. G. A., van Zadelhoff, G.-J., \& van Dishoeck, E. F. 2004, A\&A, 428, 511

Jørgensen, J. K., Bourke, T. L., Myers, P. C., et al. 2005, ApJ, 632, 973

Lommen, D., Wright, C. M., Maddison, S. T., et al. 2007, A\&A, 462, 211

Ossenkopf, V., \& Henning, T. 1994, A\&A, 291, 943

Panić, O., Hogerheijde, M. R., Wilner, D., \& Qi, C. 2008, ArXiv e-prints

Piétu, V., Dutrey, A., \& Guilloteau, S. 2007, A\&A, 467, 163
Pinte, C., Padgett, D. L., Menard, F., et al. 2008, ArXiv e-prints, 808 Pringle, J. E. 1981, ARA\&A, 19, 137

Qi, C., Ho, P. T. P., Wilner, D. J., et al. 2004, ApJ, 616, L11

Qi, C., Wilner, D. J., Calvet, N., et al. 2006, ApJ, 636, L157

Raman, A., Lisanti, M., Wilner, D. J., Qi, C., \& Hogerheijde, M. 2006, AJ, 131, 2290

Sault, R. J., Teuben, P. J., \& Wright, M. C. H. 1995, in Astronomical Data Analysis Software and Systems IV, ed. R. A. Shaw, H. E. Payne, \& J. J. E. Hayes, ASP Conf. Ser., 433, 77

Schöier, F. L., van der Tak, F. F. S., van Dishoeck, E. F., \& Black, J. H. 2005, A\&A, 432, 369

Swenson, F. J., Faulkner, J., Rogers, F. J., \& Iglesias, C. A. 1994, ApJ, 425, 286

van Kempen, T. A., van Dishoeck, E. F., Brinch, C., \& Hogerheijde, M. R. 2007, A\&A, 461, 983

van Zadelhoff, G.-J., Aikawa, Y., Hogerheijde, M. R., \& van Dishoeck, E. F. 2003, A\&A, 397, 789

Weidenschilling, S. J. 1977, MNRAS, 180, 57

Whipple, F. L. 1972, in From Plasma to Planet, ed. A. Elvius

Wichmann, R., Bastian, U., Krautter, J., Jankovics, I., \& Rucinski, S. M. 1998, MNRAS, 301, L39

Wilson, T. L., \& Rood, R. 1994, ARA\&A, 32, 191 